\title{
Deconfining by Winding: The Magnetic Monopole Vacua at High Temperatures
}

\author{
Ralf Hofmann \\ Theoretical Physics Institute, University of Minnesota, Minneapolis, MN 55455, USA
}

TPI-MINN-00/32

June 2000

\begin{abstract}
Characterizing the vacuum of a thermalized SU(3) Yang-Mills theory in the dual Ginzburg-Landau description, the possibility of topologically nontrivial, classical monopole fields in the deconfining phase is explored. These fields are assumed to be Bogomol'nyi-Prasad-Sommerfield (BPS) saturated solutions along the compact, euclidean time dimension. A corresponding, gauge invariant monopole interaction is constructed. The model passes first tests. In particular, a reasonable value for the critical temperature is obtained, and the partial persistence of nonperturbative features in the deconfining phase of SU(3) Yang-Mills theory, as it is measured on the lattice, follows naturally.
\end{abstract}

\section{INTRODUCTION}

The idea of explaining confinement in $\mathrm{SU}(\mathrm{N})$ Yang-Mills theory via the dual Meissner effect has been put forward by Mandelstam and 't Hooft in the late seventies and early eighties [1]. In this picture magnetic monopoles occur as point-like objects in three-space which are charged under the gauge group $\mathrm{U}(1)^{\mathrm{N}-1}$ constituting the maximal abelian subgroup to leave a certain gauge condition intact. The condition is that a certain matrix quantity (say the nonabelian field strength component $\mathbf{G}_{23}$ ), transforming homogenously under the gauge group, must be diagonal. It is shown that at points with a degeneracy of eigenvalues the transformation leading to the fulfillment of the gauge condition causes singularities of the colormagnetic flux, that is, magnetic monopoles. Fixing the gauge in a maximal abelian manner, one hence obtains an abelian theory of electric and magnetic charges. Working with N-1 (instead of 2(N-1)) abelian gauge fields only, the Lagrangian of the theory necessarily contains nonlocal terms which one would like to avoid [2]. The way out is the introduction of a twin set of gauge fields [2.3]. The resulting theory contains electrically and magnetically charged matter fields and sets of (N-1) electric and magnetic abelian gauge fields $\vec{A}_{\mu}$ and $\vec{B}_{\mu}$, respectively. A phenomenological self-interaction of the magnetic monopole fields is assumed to yield a spontaneously generated condensate breaking the $\mathrm{U}(1)^{\mathrm{N}-1}$ symmetry [4]. As a result, the set of gauge fields $\vec{B}_{\mu}$, interacting with the magnetic charges 円, becomes massive and the colorelectric flux between two heavy quarks of opposite color charge, separated by a distance $R$, is confined to a straight tube (string) of constant tension $\sigma$ causing a potential linearily rising with $R$. This is the celebrated confinement mechanism. The occurence of a monopole condensate, starting from first principles, has never been proven analytically. However, a set of local and non-local lattice interactions of monopole currents has been fitted to the output of Monte-Carlo lattice simulations of maximal abelian gauge fixed SU(2) in Refs. [5:7]. As a result the condensation of monopoles in the continuum limit was proven using energy-entropy balance arguments. An extension of this work to the $\mathrm{SU}(3)$ case was reported in Ref. [6].

It has been shown on the lattice in the more recent past that a thermalized SU(3) Yang-Mills theory undergoes a deconfinement phase transition at critical temperatures $T_{c}^{l a t} \sim 200-300 \mathrm{MeV}$ (see for example Ref. [11]). In the framework of the dual Ginzburg-Landau theory the deconfinement phase transition at finite temperature has been first discussed in Ref. [9]. In Ref. [10] the critical temperature $T_{c}$ of the deconfinement phase transition was determined as the point where a thermal one-loop effective potential starts possessing a trivial, absolute minimum. The analysis was based on a Higgs-like (renormalizable) self-interaction of the monopole fields. From the $T$-dependence of the monopole condensate the temperature evolutions of the vector and monopole masses $m_{B}, m_{\phi}$ were calculated which in turn determine the thermal string tension $\sigma$.

In the present work we start with a different philosophy. Based on the assumption of a gauge invariant potential for the (self-)interaction of classical monopole fields $\phi_{k}, k=1,2,3$, and considering only the $\mathrm{U}(1)^{2}$ electric and magnetic gauge fields to fluctuate, we obtain a picture for the deconfinement phase transition and quantitative results for nonperturbative features in the deconfining phase. Thereby, the potential is constructed to allow for both,

${ }^{1}$ This sector is a dual Ginzburg-Landau theory. 
topologically trivial and nontrivial $(T>0)$ BPS saturated solutions for each of the monopole fields $\phi_{k}$. As far as ground state properties are concerned, we feel that the assumption of classical monopole dynamics is justified by the observation that nonperturbative quantities like the string tension $\sigma$ could be determined reasonably well within the meanfield approximation in Refs. [4, 8].

The starting point of our investigations is the dual Ginzburg-Landau theory given by the following Lagrangian [ 四]

$$
\mathcal{L}_{D G L}=-\frac{1}{4}\left(\partial_{\mu} \vec{B}_{\nu}-\partial_{\nu} \vec{B}_{\mu}\right)^{2}+\sum_{k=1}^{3}\left\{\left|\left(i \partial_{\mu}-g \vec{\varepsilon}_{k} \cdot \vec{B}_{\mu}\right) \phi_{k}\right|^{2}-V_{k}\left(\phi_{k}, \bar{\phi}_{k}\right)\right\} .
$$

This theory decribes the magnetic sector emerging from $\mathrm{SU}(3)$ Yang-Mills theory due to maximal-abelian gauge fixing [4]. In contrast to the electric, abelian gauge group the magnetic $\mathrm{U}(1)^{2}$ (with gauge field $\vec{B}_{\mu}=\left(B_{\mu}^{3}, B_{\mu}^{8}\right)$ ) is believed to be spontaneously broken by nonvanishing VEVs of the monopole fields $\phi_{k}$. In Eq. (11) $g \vec{\varepsilon}_{i}$ denote the effective magnetic charges with $\vec{\varepsilon}_{1}=(1,0), \vec{\varepsilon}_{2}=(-1 / 2,-\sqrt{3} / 2), \vec{\varepsilon}_{3}=(-1 / 2, \sqrt{3} / 2)$, and the fields $\phi_{k}$ satisfy the constraint 叫

$$
\sum_{k=1}^{3} \arg \phi_{k}=0
$$

The potential

$$
V \equiv \sum_{k=1}^{3} V_{k}\left(\phi_{k}, \bar{\phi}_{k}\right)
$$

is introduced phenomenologically to account for the self-interaction of the monopole fields $\phi_{k}$. For the sake of renormalizability and the desired feature of spontaneous breaking of the magnetic gauge symmetry it is usually chosen to be of the Higgs form

$$
\lambda \sum_{k=1}^{3}\left(\bar{\phi}_{k} \phi_{k}-v^{2}\right)^{2}
$$

Corresponding to Eq. (何), the one-loop effective potential at finite temperature, calculated in euclidean space-time with compact time dimension of size $\beta \equiv 1 / T$, possesses a global minimum of vanishing condensate for $T>T_{c} \sim 500$ $\mathrm{MeV}$ [10]. This result is probably due to the sole consideration of quadratic fluctuations, since there are indications that for example a quartic term is parametrically not small (In one-loop approximation the coupling constant for this term is $\lambda \sim 25$ !). In order to bring $T_{c}$ down to values compatible with lattice experiments $\left(T_{c}^{\text {lat }} \sim 200-300 \mathrm{MeV}\right)$ the authors of Ref. [10] had to introduce a $T$-dependence of $\lambda$ by hand.

Here, we postulate that the occurence of a phase transition is due to the thermalized system admitting topologically nontrivial ground state solutions in the monopole sector. Accordingly, we introduce a gauge invariant (self-)interaction for the classical fields $\phi_{k}$. To construct the potential we furthermore assume that the relevant solutions to the corresponding field equations are BPS saturated. This is in analogy to the case of instantons in non-gauge-fixed Yang-Mills theories, where the condition of self-duality is equivalent to the saturation of the Bogomoln'yi bound for the euclidean action [14]. Since at $T=0$ the ground state solution is a constant and at $T>0$ changes are induced by the finite size of the euclidean, compact time dimension it is reasonable to assume that the topologically nontrivial structure of the vacuum above $T_{c}$ is brought about by a sole dependence on euclidean time $\tau$. In Refs. [12, 13] it was shown in the framework of supersymmetric theories that for a scalar sector to admit nontrivial BPS saturated solutions along a compact dimension the target space must possess noncontractable cycles. In Ref. [15] a generalized Wess-Zumino model, possessing a discrete set of $N+1$ nonzero vacua (proportional to the $N+1$ unit roots), was investigated. These vacua are interpolated by BPS saturated, periodic solutions along the compact dimension. As in Ref. [15], we concentrate on the case, where there is one single pole at $\phi_{k}=0$ on the rhs of the BPS equation. Since the monopole sector of the theory should $\mathbf{1}$ ) admit nonzero, constant solutions at $T=0,2$ ) be gauge invariant under the magnetic $\mathrm{U}(1)^{2}$, and 3) admit nontrivial, periodic, BPS saturated solutions for $T>0$ the potential is given as 15.

$$
V_{k}\left(\phi_{k}, \bar{\phi}_{k}\right)=\lim _{N \rightarrow \infty}\left\{\frac{\Lambda^{6}}{\bar{\phi}_{k} \phi_{k}}+\kappa^{2} \Lambda^{-2(N-2)}\left(\bar{\phi}_{k} \phi_{k}\right)^{N}-2 \kappa \Lambda^{5-N} \frac{1}{\bar{\phi}_{k} \phi_{k}} \operatorname{Re} \phi_{k}^{N+1}\right\}
$$


Thereby, $\Lambda$ is a mass-parameter, and $\kappa$ is some dimensionless coupling constant. Considering the rhs of Eq. (河) at finite $N$, the potential explicitely breaks the magnetic $\mathrm{U}(1)^{2}$ gauge symmetry

$$
\vec{B}_{\mu} \rightarrow \vec{B}_{\mu}+1 / g \partial_{\mu} \vec{\theta}(x), \quad \phi_{k} \rightarrow \mathrm{e}^{-i \overrightarrow{\varepsilon_{k}} \cdot \vec{\theta}(x)} \phi_{k}
$$

down to $\mathrm{Z}_{N+1}^{2}$ due to the term $\operatorname{Re} \phi_{k}^{N+1}$. The vacua in this case are given as 15

$$
\frac{\Lambda}{\kappa^{1 /(N+1)}}\left(\mathrm{e}^{\frac{2 \pi \alpha_{1}}{N+1} i}, \mathrm{e}^{\frac{2 \pi \alpha_{2}}{N+1} i}, \mathrm{e}^{\frac{2 \pi \alpha_{3}}{N+1} i}\right), \quad\left(\alpha_{k} \in \mathbf{Z},-N \leq \alpha_{k} \leq N, \sum_{k=1}^{3} \alpha_{k}=0\right) .
$$

Only in the limit of large $N$ is the gauge symmetry restored. For the purpose of qualitative illustration Fig. 1 indicates the potential $V_{k}$ for $\kappa=1, N=500$, and dimensionless $\Lambda=0.5$.

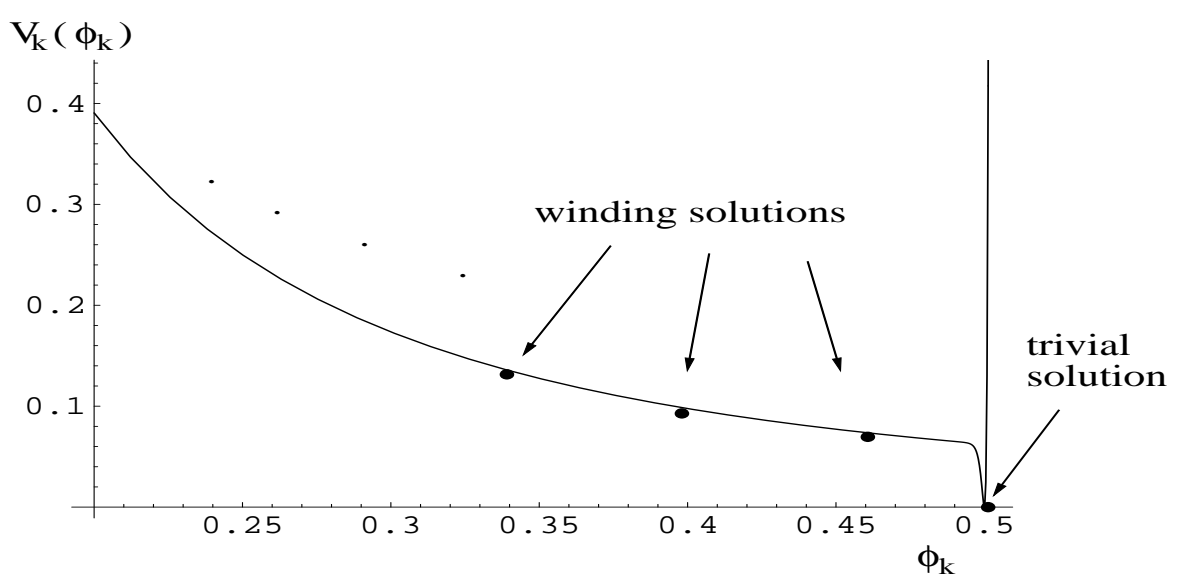

FIG. 1. The monopole potential $V_{k}$ as a function of real $\phi_{k}$ for $\kappa=1, \Lambda=0.5$, and $N=500$. Indicated are the potential energy densities of topologically trivial and nontrivial solutions to the BPS equations.

The BPS equations, corresponding to the potential of Eq. (5), read

$$
\partial_{\tau} \phi_{k}=\bar{V}_{1 / 2}^{k}\left(\bar{\phi}_{k}\right), \partial_{\tau} \bar{\phi}_{k}=V_{1 / 2}^{k}\left(\phi_{k}\right) .
$$

The rhs are only fixed up to phase factors $\mathrm{e}^{i \delta}, \mathrm{e}^{-i \delta}$, respectively. From Eq. (5) we have for periodic solutions

$$
V_{1 / 2}^{k}\left(\phi_{k}\right)=\left\{\begin{array}{cc} 
\pm i \frac{\Lambda^{3}}{\phi_{k}}, & \left(\left|\phi_{k}\right|<\Lambda\right) \\
0, & \left(\left|\phi_{k}\right|=\Lambda\right) \\
\infty, & \left(\left|\phi_{k}\right|>\Lambda\right)
\end{array}\right.
$$

The paper can be outlined as follows: In the next section we briefly review what has to be known about BPS saturated winding solutions along a compact dimension 12, 13, 15. We device a (not unique) label for the winding vacua, and we write down the gauge function to transform a winding vacuum to unitary gauge. Section 3 investigates the transition to the deconfined phase. The critical temperature is estimated by assuming thermal equilibrium between the winding vacuum and an ideal gluon gas. The consequences of winding vacua in the deconfined phase are examined in Section 4. The temperature evolutions of the monopole condensate (and thereby that of $m_{B}$ ) and the string tension are elucidated. Section 5 summarizes the results, comments on the approximations made, and gives the conclusions.

\section{BPS SATURATED WINDING SOLUTIONS}

For the case $\left|\phi_{k}\right|<\Lambda$ periodic solutions $\left(\phi_{k}(0)=\phi_{k}(\beta)\right)$ to Eqs. (8) subject to Eq. (9) have been discussed in Refs. 12, 13. within the framework of supersymmetric theories. For a compact, euclidean time dimension of length $\beta \equiv 1 / T$ the set of topologically distinct solutions is

$$
\phi_{k}^{n_{k}}(\tau)=\sqrt{\frac{\Lambda^{3} \beta}{2\left|n_{k}\right| \pi}} \mathrm{e}^{2 n_{k} \pi i \frac{\tau}{\beta}}, \quad\left(n_{k} \in \mathbf{Z}\right) .
$$


Thereby, the sign of $n_{k}$ corresponds to the choice of phase in the BPS equations. Due to the phase constraint of Eq. (2) the sets of solutions $\left(\phi_{1}^{n_{1}}, \phi_{2}^{n_{2}}, \phi_{3}^{n_{3}}\right)$ can be labeled by the integers $n_{1}$ and $m$, which are both odd or even. The winding numbers $n_{2}, n_{3}$ are then given as $-n_{1} / 2 \mp m / 2$, respectively. With the gauge transformation of Eq. (6) the function $\vec{\theta}_{n_{1}, m}(\tau)$, transforming to the unitary gauge $\operatorname{Re} \phi_{k}(\tau)>0, \operatorname{Im} \phi_{k}(\tau)=0$, reads

$$
\vec{\theta}_{n_{1}, m}(\tau)=\frac{2 \pi}{\beta} \tau\left(\begin{array}{c}
n_{1} \\
\frac{m}{\sqrt{3}}
\end{array}\right)
$$

Note that this nonperiodic function leaves the periodicity of the gauge field $\vec{B}_{\mu}$ intact.

\section{DECONFINEMENT PHASE TRANSITION}

Eqs. (8) admit the solution $\phi_{k}^{0} \equiv \Lambda$ with winding number and energy density zero, and the winding solutions of the previous section. We identify the set $\left(\phi_{1}^{0}, \phi_{2}^{0}, \phi_{3}^{0}\right)$ with the confining vacuum at low temperatures. In this regime the spectrum consists of glueballs with masses that are much larger than the prevailing temperatures [8]. The thermal equilibrium between the vacuum "medium" and its excitations is essentially realized at pressure zero, which indeed is observed on the lattice $[17$. Above the deconfinement phase transition the ground state "medium" readily emits and absorbs (almost) free gluons under the influence of the heat bath like a black body emits and absorbs photons. We identify the genuine winding set of lowest potential energy density 2 , represented by $\left(n_{1}, m\right)=(2,0)$, with the ground state just above the deconfinement transition. In this picture the confining vacuum is a perfect thermal insulator up to the transition, where its structure drastically changes by the absorption of an amount of latent heat per volume [17] equal to the gap $\Delta \varepsilon^{(2,0)}$ between the potential $V$ of the zero winding and the lowest genuine winding set. We estimate the critical temperature $T_{c}$ of this transition by assuming the deconfining vacuum to behave like an incompressible, static fluid with traceless energy-momentum-tensor [16] which is in thermal equilibrium with an ideal gas of gluons. In this case we have the equation of state

$$
\varepsilon=3 p
$$

for pressure $p$ and energy density $\varepsilon$ for both the vacuum (vac) and the gluon gas (gg). The equilibrium condition of equal pressures,

$$
p_{v a c}=p_{g g}
$$

then takes the following form [16]

$$
\Delta \varepsilon^{(2,0)}=\frac{8}{15} \pi^{2} T_{c}^{4}
$$

where

$$
\Delta \varepsilon^{(2,0)}=2 \times \frac{\Lambda^{6}}{\bar{\phi}^{1} \phi^{1}}+\frac{\Lambda^{6}}{\bar{\phi}^{2} \phi^{2}}=8 \pi \Lambda^{3} T_{c}
$$

Using Eqs. (14),(15), we obtain

$$
T_{c}=\left(\frac{15}{\pi}\right)^{\frac{1}{3}} \Lambda \sim 1.68 \Lambda .
$$

Adopting the value $\Lambda=0.126 \mathrm{GeV}$ for the monopole condensate at $T=0$ from Refs. [8, 10], Eq. (16) yields $T_{c}=0.212$ $\mathrm{GeV}$. This is compatible with the lattice results of $T_{c}=0.2-0.3 \mathrm{GeV}$.

\footnotetext{
${ }^{2}$ By "genuine" we mean that each of the solutions $\phi_{k}$ is winding. Note, however, that there are also semi-winding sets, for example $\left(n_{1}, m\right)=(1,1)$, which contain two winding fields and one field of winding number zero. Since none of the monopole fields $\phi_{k}$ should be singled out it is natural to assume that either all $\phi_{k}$ are winding or none at all.
} 


\section{THE DECONFINING PHASE}

For a set of solutions with

$$
l \equiv \sum_{k=1}^{3}\left|n_{k}\right|
$$

the equilibrium condition of Eq. (13) yields the following dependence of the temperature at equilibrium $T_{l}$ on $l$

$$
T_{l}=\left(\frac{15 l}{4 \pi}\right)^{\frac{1}{3}} \Lambda
$$

Modulo degeneracy the number of equilibrium states per temperature interval $\rho(T)$ thus grows quadratically with increasing $T_{l}$

$$
\rho(T) \sim \frac{d l}{d T_{l}}=\frac{4 \pi}{5} \frac{T_{l}^{2}}{\Lambda^{3}} .
$$

For temperatures $T \neq T_{l}$ the system is thermodynamically unstable within the above approximations, and hence it must evolve to the nearest equilibrium point. Let us now look at the change of the average monopole condensate

$$
\phi_{a v}^{\left(n_{1}, m\right)} \equiv \frac{1}{3} \sum_{k=1}^{3} \phi_{k}^{n_{k}}
$$

across the transition from the confining to the deconfining phases. Transforming the winding solution of Eq. (10) to the unitary gauge by means of Eqs. (6), (11), we obtain the following expression for the individual condensate, corresponding to winding $n_{k}$ and contributing to an $l$-winding vacuum

$$
\phi_{k}^{n_{k}}=\frac{\Lambda^{3 / 2}}{\sqrt{2 \pi\left|n_{k}\right| T_{l}}}=\frac{\Lambda}{\sqrt{\left(30 \pi^{2} l\right)^{1 / 3}\left|n_{k}\right|}} .
$$

For the configuration $\left(n_{1}, m\right)=(2,0)$ we obtain

$$
\phi_{a v}^{(2,0)} \sim 0.28 \Lambda
$$

In Fig. 2 the evolution of the average monopole condensate is depicted up to $T \sim 0.41 \mathrm{GeV}$. The arrows point at states of thermal equilibrium. The corresponding representatives, labeled by $\left(n_{1}, m\right)$, evolve into nonequilibrium until the next equilibrium state becomes available.

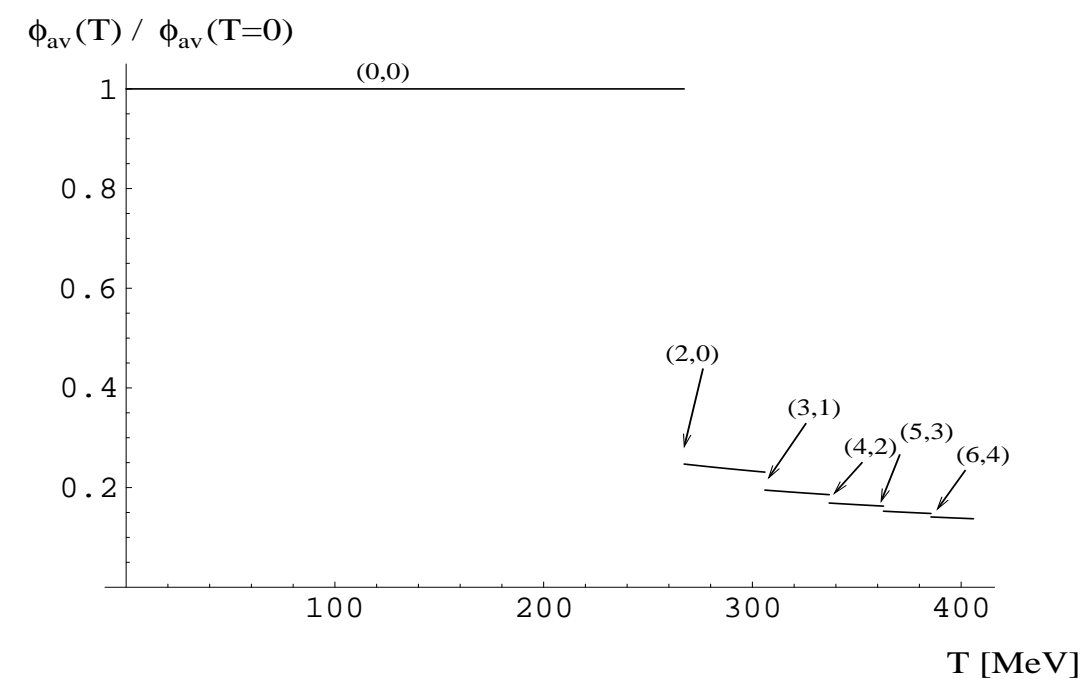

FIG. 2. The average monopole field $\phi_{a v}(T)$ in units of $\phi_{a v}(T=0)$. For further explanation see text. 
In unitary gauge the monopole fields are real, and the spontaneous breakdown of the $\mathrm{U}(1)^{2}$ magnetic gauge symmetry then transparently generates a mass $m_{B}$ for the field $\vec{B}_{\mu}$ which scales linearly with the average monopole condensate:

$$
m_{B}^{\left(n_{1}, m\right)}=\sqrt{3} g \phi_{a v}^{\left(n_{1}, m\right)} .
$$

In Refs. [8, 10 the value $m_{B} \sim 0.5 \mathrm{GeV}$ was determined at $T=0$, yielding a magnetic gauge coupling of $g=2.3$.

How does the string tension $\sigma$ evolve? An analytical expression for $\sigma$ in terms of $g, m_{B}$, and $m_{\phi}$ was derived in Ref. [8] by calculating the potential between two heavy and static quarks of opposite color charge, separated by a distance $R$. Thereby, an effective Lagrangian for the dynamics of the electric $U(1)^{2}$ gauge field $\vec{A}_{\mu}$ is obtained by integrating out the massive, magnetic vector field $\vec{B}_{\mu}$ and by assuming that the monopole fields do not fluctuate about their VEVs. Applying convenient gauge fixing terms and introducing external, static color currents, which couple to $\vec{A}_{0}$, one obtains an effective action. From this action one extracts the potential energy $V_{\bar{q} q}(R)$ of the static sources. It assumes the following form

$$
V_{\bar{q} q}(R)=-\frac{\vec{Q}^{2}}{4 \pi}\left(\frac{\mathrm{e}^{-m_{B}^{2} R}}{R}+\frac{m_{B}^{2}}{2} \ln \left[1+\frac{m_{\phi}^{2}}{m_{B}^{2}}\right] R\right),
$$

where $\vec{Q}=Q_{3}^{2}+Q_{8}^{2}=e^{3} / 3$ 近. From Eq. (24) $\sigma$ can be read off as

$$
\sigma=\frac{2 \pi m_{B}^{2}}{3 g^{2}} \ln \left[1+\frac{m_{\phi}^{2}}{m_{B}^{2}}\right] .
$$

Thereby, we have used the relation $e \cdot g=4 \pi$ linking the magnetic with the electric charge. Since static test charges were assumed finite temperature does not necessitate any new considerations, and thus $\sigma$ is still given by Eq. (25) with an implicit $T$-dependence via $m_{B}(T)$ and $m_{\phi}(T)$.

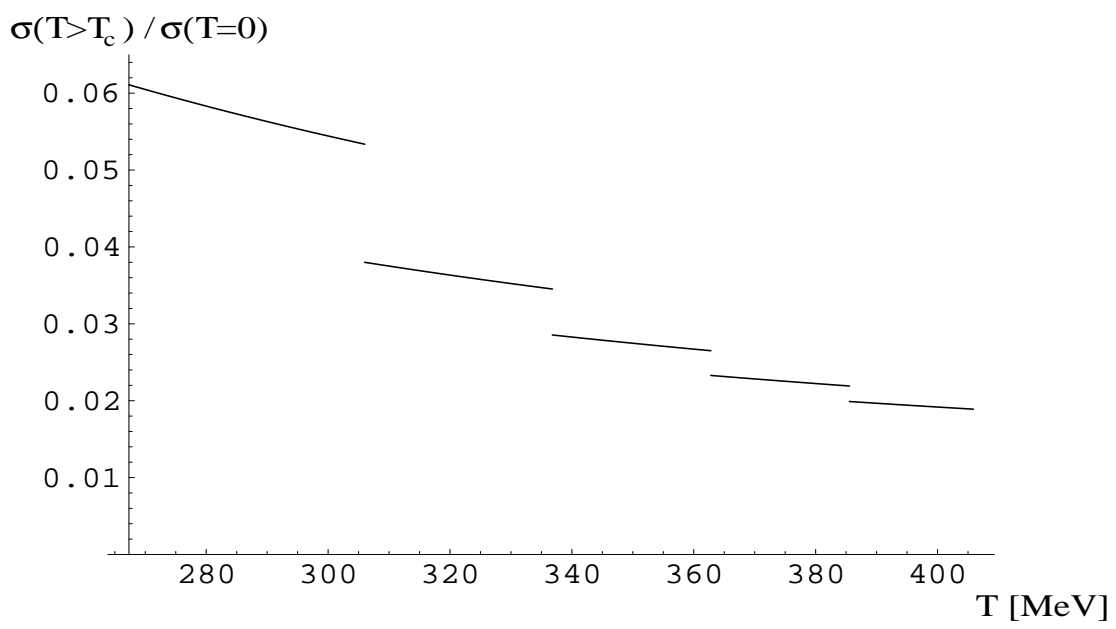

FIG. 3. The string tension $\sigma\left(T>T_{c}\right)$ in units of $\sigma(T=0)$ in the deconfining phase.

The appearance of the monopole mass $m_{\phi}>m_{B}$ under the logarithm in Eq. (25) is due to its role as an ultraviolet cutoff for the integration over transverse momenta in the expression for the linear part of the potential in Eq. (24). This is justified by the observation that $m_{B} \sim 0$ inside the flux tube of radius $\rho \sim m_{\phi}^{-1}$ in a type II dual superconductor (see Ref. [10] and Refs. therein). Since we work with classical fields $\phi_{k}$ we cannot asign a mass to the fluctuations of the monopole fields about the background of a classical ground state. We may, however, assume that $\rho^{-1}$ scales with temperature in the same way as $m_{B}$ does. Since the dependence of $\sigma$ on the cutoff is logarithmic the result is not dramatically sensitive to the exact $T$ dependence of $\rho^{-1}$, which we will make explicit by distinguishing the following cases:

(a) $\rho^{-1}(0)=\rho^{-1}(T)=1.26 \mathrm{GeV}[\mathbb{8}] \Rightarrow$

$$
\frac{\sigma\left(T_{c}\right)}{\sigma(0)} \sim(0.28)^{2} \frac{\ln \left(1+(1.26)^{2} /(0.5 \times 0.28)^{2}\right)}{\ln \left(1+(1.26)^{2} /(0.5)^{2}\right)} \sim 0.19
$$


(b) $\rho^{-1}(T) \propto m_{B}(T) \Rightarrow$

$$
\frac{\sigma\left(T_{c}\right)}{\sigma(0)} \sim(0.28)^{2} \sim 0.08
$$

Hence, the difference roughly amounts to a factor 2. Assuming case (b), we show the $T$-dependence of $\sigma(T) / \sigma(0)$ for the deconfining phase in Fig. 3.

\section{SUMMARY AND DISCUSSION}

We considered a thermalized dual Ginzburg-Landau theory modelling hot SU(3) Yang-Mills theory. From the postulate that in contrast to the confining phase the ground state of the deconfining phase is characterized by topologically nontrivial, BPS saturated solutions to the classical equations of motion of the monopole sector we deviced the corresponding, gauge invariant interaction. As a consequence, the average monopole field undergoes a drastic decrease to about $1 / 4$ of its zero temperature value across the phase boundary. Scaling linearly with the monopole condensate, the same applies to the mass of the magnetic vector fields generated by the spontaneous breaking of the magnetic $\mathrm{U}(1)^{2}$ gauge symmetry. Working with the zero temperature value for the monopole condensate of Refs. [8, 10, we obtain a rather reasonable critical temperature $T_{c} \sim 212 \mathrm{MeV}$ in contrast to the effective potential calculation of Ref. [10], where $T_{c} \sim 500 \mathrm{MeV}$, and the more realistic value of $T_{c} \sim 200 \mathrm{MeV}$ was obtained by introducing an ad hoc $T$-dependence of the dimensionless coupling constant $\lambda$.

Depending on the assumption about the $T$-dependence of the flux tube radius $\rho$ the string tension $\sigma$ decreases to about $1 / 12 . .1 / 5$ of its value at $T=0$ across the phase boundary which is compatible with lattice measurements [17]. To determine $T_{c}$ we assumed a free gluon gas which is a rough approximation due to the fact that nonperturbative features do persist to a sizable extend in the deconfined phase. This is indicated by nonvanishing values of the monopole condensate $\phi_{a v}$ and the string tension $\sigma$. At higher temperatures the ideal gas approximation should become better, and the model predicts a slow decrease of these quantities. For example, at $T \sim 1.5 T_{c}$ we have

$$
\frac{\phi_{a v}\left(T=1.5 T_{c}\right)}{\phi_{a v}(T=0)} \sim \frac{1}{2} \times \frac{\phi_{a v}\left(T=T_{c}\right)}{\phi_{a v}(T=0)} .
$$

On the lattice and in effective potential calculations the deconfinement phase transition has been determined to be of first order [10, 17, 18]. In contrast, SU(2) Yang-Mills theory exhibits a second order phase transition [18]. The model of the present work can be easily adapted to the dual Ginzburg-Landau theory describing maximal abelian gauge fixed SU(2) Yang-Mills theory. The qualitative results would then be the same. Hence, the model is too coarse to resolve the order of the phase transition. However, it gives a reason for the residual, nontrivial structure of monopole condensation in $\mathrm{SU}(2)$ and $\mathrm{SU}(3)$ Yang-Mills theories at high temperatures, it predicts a reasonable value of the critical temperature, and it incorporates the low temperature limit in the form of a constant solution to the BPS equations. The existence of a nontrivial structure of monopole condensation above the phase transition has been pointed out in Ref. [19] a long time ago based on lattice investigations.

To conclude, we showed that in a thermalized, generalized dual Ginzburg-Landau theory, describing the magnetic part of SU(3) Yang-Mills theory subject to maximal abelian gauge fixing, the postulate of nontrivial, BPS saturated ground state solutions of the magnetic monopole sector in the deconfining phase leads to acceptable results. In particular, the partial conservation of nonperturbative features in the high temperature regime is a natural consequence in this picture.

\section{ACKNOWLEDGMENTS}

The author would like to thank V. Eletsky and M. Pospelov for stimulating discussions at an early stage of this work. Continuous, useful conversations with T. ter Veldhuis are gratefully acknowledged. This work was funded by a fellowship of Deutscher Akademischer Austauschdienst (DAAD).

[1] S. Mandelstam, Phys. Rep. C23, 245 (1976). G. 't Hooft, Nucl. Phys. B190, 455 (1981). 
[2] K. Bardakci and S. Samuel, Phys. Rev. D18, 2849 (1978).

[3] D. Zwanziger, Phys. Rev. D3, 880 (1970).

[4] T. Suzuki, Prog. Theor. Phys. 80, 929 (1988); 81, 752 (1989).

S. Maedan and T. Suzuki, Prog. Theor. Phys. 81, 229 (1989).

[5] H. Shiba and T. Suzuki, Phys. Lett. B351, 519 (1995).

[6] K. Yamagishi, S. Kitahara and T. Suzuki, JHEP 0002, 012 (2000).

[7] M. N. Chernodub, S. Fujimoto, S. Kato, M. Murata, M. I. Polikarpov and T. Suzuki, hep-lat/0006025, to appear in Phys. Rev. D.

[8] H. Suganuma, S. Sasaki and H. Toki, Nucl. Phys. B435, 207 (1995).

[9] H. Monden, T. Suzuki and Y. Matsubara, Phys. Lett. B294, 100 (1992)

[10] H. Ichie, H. Suganuma and H. Toki, Phys. Rev. D52, 2944 (1995).

[11] T. Hashimoto et al., Phys. Rev. D42, 620 (1990).

[12] G. Dvali and M. Shifman, Phys. Lett. B454, 277 (1999).

[13] X. Hou, A. Losev and M. Shifman, Phys. Rev. D61, 085005 (2000).

[14] T. Schafer and E. V. Shuryak, Rev. Mod. Phys. 70, 323 (1998).

[15] R. Hofmann, hep-th/0004178, to appear in Phys. Rev. D.

[16] J. Cleymans, E. Nykänen and E. Suhonen, Phys. Rev. D33, 2585 (1986).

[17] B. Beinlich et al., Eur. Phys. J. C6, 133 (1999).

[18] F. Karsch, "THE DECONFINEMENT TRANSITION IN FINITE TEMPERATURE LATTICE GAUGE THEORY", Invited talk given at the 'Enrico Fermi' Int. School of Physics, Varenna, Italy, Jun 26-Jul 6, 1984.

[19] M. L. Laursen and G. Schierholz, Z. Phys. C38, 501 (1988). 